combines self-sampling from different anatomical sites, automated data input and sample management including LIS based registration of the sample tubes. The Panther ${ }^{\circledR}$ protocol combines target detection of different STI pathogens. The results are transferred directly into the LIS for validation and reporting. The sample tubes are archived including an electronic place marker for subsequent investigation.

Results The workflow enables us to proceed up to 600 samples in a 10 hours working day. We handle 250-500 samples per day. During the German MSM screening study 2018 we investigated 6900 additional samples resulting in 27600 analytical data points and more than 300.000 sociodemographic information within 3 month. Analytical result data were continuously linked to collected questionnaire information on sociodemographics (e.g. clinical symptoms, sexual behavior, PrEP-use, etc.). Result reports were delivered every day and cumulative data sets were exported from LIS for epidemiological evaluation every week.

Conclusion We have adapted a workflow for laboratory requirements of epidemiologic STI studies. This is essential for efficient data management processes. By integrating molecular instruments into a combined sample management process automation has become a fundamental principle in laboratory processes but is also indispensable for conducting STI investigations. We have already implemented the process successfully during the 'MSM Screening Study 2018' and the ongoing 'BRAHMS-Study 2018'.

Disclosure No significant relationships.

\section{P026 FREQUENCY OF STD TESTING SERVICES AMONG COMMERCIALLY-INSURED PATIENTS WITH HIGH RISK SEXUAL BEHAVIORS}

${ }^{1}$ Sagar Kumar*, ${ }^{2}$ Chirag Patel, ${ }^{2}$ Guoyu Tao. ${ }^{1}$ Centers for Disease Control and Prevention, Division of STD Prevention, Atlanta, USA; ${ }^{2}$ Centers for Disease Control and Prevention, Atlanta, USA

\subsection{6/sextrans-2019-sti.235}

Background High-risk sexual behavior (HRSB) is associated with an increased burden of sexually transmitted diseases (STDs); therefore, CDC recommends those at high risk are screened more frequently than persons without HRSB. Because providers are able to document patients as having 3 types of HRSB: heterosexual, homosexual, or bisexual, using the International Classification of Disease Tenth Revision (ICD-10), this study assessed STD/HIV screening frequencies among patients with HRSB diagnoses.

Methods A large commercial claims database for outpatient visits for 2016 was analyzed. We included in the analysis patients diagnosed with any HRSB based on ICD-10 codes. The initial diagnosis for HRSB was considered as the index date for each patient. Testing frequencies for chlamydia, gonorrhea, syphilis, and HIV were assessed by 3 types of HRSB. For those diagnosed with HRSB from January 1-June 30, 2016, an additional 6 month follow-up testing period was also assessed.

Results 52,160 patients were diagnosed with HRSB in 2016: 90.3\% were heterosexual, $7.7 \%$ homosexual, and $2.1 \%$ bisexual HRSB. Testing for chlamydia, gonorrhea, syphilis, and HIV was $65.3 \%, 65.2 \%, 38.1 \%$, and $43.6 \%$ for heterosexual, $49.9 \%, 49.8 \%, 51.5 \%$, and $57.8 \%$ for homosexual, and $57.7 \%, 56.6 \%, 41.6 \%$, and $48.2 \%$ for bisexual HRSB patients at the initial diagnosis date. $35.5 \%, 20.9 \%$, and $36.5 \%$ of heterosexual, homosexual, and bisexual HRSB patients were found to have follow-up claims within 6 months after the index date, respectively. Of those who had follow-up claims, follow-up testing for chlamydia, gonorrhea, syphilis, and HIV was $77.2 \%, 77.6 \%, 50.6 \%$, and $56.4 \%$ for heterosexual, $71.8 \%, 72.7 \%, 74.5 \%$, and $80.4 \%$ for homosexual, and $69.7 \%, 71.1 \%, \quad 58.2 \%$, and $66.7 \%$ for bisexual HRSB patients. STD/HIV follow-up testing rates were higher among patients who were screened at the index date than those who were not.

Conclusion STD/HIV screening and follow-up screenings among HRSB patients are sub-optimal. Most HRSB patients do not have timely follow-up visits.

Disclosure No significant relationships.

\section{P027 SAFETY AND EFFECTIVENESS OF SAME-DAY SCREENING AND TREATMENT AMONG YOUTH IN LOS ANGELES, CALIFORNIA}

${ }^{1}$ Erin Keizur*, ${ }^{2}$ Wilson Ramos, ${ }^{2}$ Maryann Koussa, ${ }^{2}$ Mary Jane Rotheram-Borus, ${ }^{3}$ eeffrey Klausner, ${ }^{2}$ Atn Atn-Cares. ${ }^{1}$ UCLA- David Geffen School of Medicine, Infectious Diseases, Los Angeles, USA; ${ }^{2}$ UCLA-David Geffen School of Medicine, Psychiatry and Biobehavior, Los Angeles, USA; ${ }^{3}$ UCLA-David Geffen School of Medicine, Infectious Diseases, Los Angeles, USA

\subsection{6/sextrans-2019-sti.236}

Background Chlamydia trachomatis (CT) and Neisseria gonorrhoeae (NG) are the most common sexually transmitted infections (STIs). Lesbian, gay, bisexual, transgender, queer (LGBTQ) and homeless youth often report risk-taking behaviors such as higher number of sex partners and condom-less sex, yet they have decreased access to healthcare services. Providing treatment following testing can reduce the transmission of infection and the risk of developing complications. As part of a large, adolescent prevention study, we provided same-day $\mathrm{CT} / \mathrm{NG}$ testing and treatment for participants.

Methods We recruited 578 persons aged 12-24 years from homeless shelters, LGBTQ organizations, and community health centers in Los Angeles, California beginning May 2017. Participants received point-of-care pharyngeal, rectal, and urethral/vaginal CT/NG testing using the Cepheid GeneXpert. Beginning March 2018, after a positive CT or NG test result, participants were offered a same-day treatment pack. Participants were additionally offered treatment packs for their sex partners. We measured the proportion of participants who received treatment within 3 months of testing, the median time-to-treatment, and the number of expedited partner therapy packs taken by participants between March 2018 December 2018.

Results Before offering same-day treatment for CT/NG infection, only $35 \%(15 / 43)$ of participants had confirmed treatment. After implementing the same-day treatment packs, $76 \%$ $(42 / 55)$ of participants received treatment $(\Delta=41 \%$, p-value $<0.001)$. Of those receiving treatment, median time to treatment was 10 days (range 1-88 days) before, and 1 day (range $0-62)$ after $(\Delta=9$ days, p-value $=0.007)$. Overall, $35 \%(25 /$ 78) of participants took 36 partner therapy packs (median $=1$ pack, range 0 - 3 packs). There were no reported adverse side effects.

Conclusion Providing same day STI testing and treatment to high-risk youth is safe, feasible, and can increase the proportion of individuals receiving timely treatment.

Disclosure No significant relationships. 